\title{
Diminished Top-Down Control Underlies a Visual Imagery Deficit in Normal Aging
}

\author{
Jonathan Kalkstein, Kristen Checksfield, Jacob Bollinger, and Adam Gazzaley \\ W.M. Keck Center For Integrative Neurosciences, Departments of Neurology, Physiology and Psychiatry, University of California, San Francisco, San \\ Francisco, California 94158-2240
}

Mental imagery is involved in a wide variety of cognitive abilities, including reasoning, spatial navigation, and memory. Cognitive aging is associated with impairments in these abilities, suggesting that diminished fidelity of mental images in older adults may be related to diverse cognitive deficits. However, an age-related deficit in mental imagery and its role in memory impairment is still a matter of debate. Previous human fMRI studies demonstrated that visual imagery activates representations in category-selective visual cortex via topdown control mechanisms. Here, we use fMRI to show that normal aging is associated with diminished selectivity of visual cortex activation during visual imagery, with a corresponding reduction in the selectivity of functional connections between prefrontal cortex and visual cortices. Moreover, a relationship between reduced imagery selectivity and visual memory in older adults was established. These results reveal that aging disrupts neural networks that subserve mental imagery and offers evidence of this as a factor in age-related memory decline.

\section{Introduction}

Impairment in mental imagery in older adults is supported by behavioral studies that reveal a decline in the generation, maintenance, scanning, manipulation, and rotation of mental images (Craik and Dirkx, 1992; Dror and Kosslyn, 1994; Iachini et al., 2005; De Beni et al., 2007; Saimpont et al., 2009). However, these results rely either on self-reports of imagery strength, a method that is biased by subjective factors, or behavioral responses that require a manipulation of imagined items in mind, an approach that is complicated by the involvement of additional neural processes. For these reasons, an empirical evaluation of neurophysiological markers of mental imagery in older adults is critical for understanding the impact of aging on the imagery process itself and its relationship with other facets of cognitive aging.

Functional neuroimaging has revolutionized the study of visual imagery by establishing a neurophysiological basis for this elusive process. Studies have revealed that areas of visual association cortex (VAC) that activate selectively during the perception of visual stimuli from a particular category also activate with similar selectivity when imagining stimuli from that same category (Goebel et al., 1998; O'Craven and Kanwisher, 2000). Thus, the degree to which imagery selectively engages specialized regions of VAC provides an objective neurophysiological correlate of the fidelity of visual images.

\footnotetext{
Received June 23, 2011; revised Aug. 11, 2011; accepted Aug. 29, 2011

Author contributions: J.K. and A.G. designed research; J.K. and K.C. performed research; J.K., J.B., and A.G. contributed unpublished reagents/analytic tools; J.K., K.C., and A.G. analyzed data; J.K. and A.G. wrote the paper.

This work was supported by NIH Grant 5R01AG030395 (A.G.). We thank Ezequiel Morsella and members of the Gazzaley Lab for their insightful comments.

The authors declare no competing financial interests.

Correspondence should be addressed to Adam Gazzaley, UCSF-Mission Bay, Genentech Hall, Room N474, 600 16th Street, Box 2240, San Francisco, CA 94158-2240. E-mail: Adam.gazzaley@ucsf.edu.

DOI:10.1523/JNEUROSCI.3209-11.2011

Copyright $\odot 2011$ the authors $\quad 0270-6474 / 11 / 3115768-07 \$ 15.00 / 0$
}

During imagery, the selective activation of cortical representations occurs in the absence of bottom-up input, and so its generation has been attributed to top-down control mechanisms. Consistent with this notion, visual imagery has been shown to activate areas of the prefrontal cortex (PFC) important in top-down modulation, such as the middle frontal gyrus (Kosslyn et al., 1997; Ishai et al., 2000), and functional connectivity analysis has revealed that network communication between PFC and VAC is associated with visual imagery (Mechelli et al., 2004). Moreover, cortical volumes of PFC regions correlate with behavioral measures of imagery in older adults (Briggs et al., 1999). We hypothesized that if an age-related deterioration in the imagery process exists, it would manifest itself via alterations in category-selective VAC activation and functional connectivity between the PFC and VAC.

To explore these hypotheses, fMRI data were collected in an experiment using a novel event-related paradigm that involved healthy younger $(n=16$; age range, $19-34$ years $)$ and older $(n=19$; age range, 62- 83 years) adults viewing and imagining two types of stimuli in separate blocks (faces and moving dots). During perception and imagery in younger adults, these stimuli have previously been shown to selectively activate distinct areas of VAC: fusiform face area (FFA) for faces (O'Craven and Kanwisher, 2000) and cortical area V5/human MT+ for motion (Goebel et al., 1998) (Fig. 1). Using these stimuli in the study allowed us to assess the presence of agerelated alterations in imagery-induced cortical representations in both ventral and dorsal visual pathways. Additionally, we administered an evaluation of visual memory to older adults outside of the scanner to test for a relationship between age-related changes in visual memory and imagery.

\section{Materials and Methods}

Participants. Sixteen healthy younger adults (age $26.6 \pm 4.34$ years; range 19-34 years; eight female) and 19 healthy older adults (age $69.8 \pm 6.0$ years; range $62-83$ years, 11 female) gave written informed consent to 




Figure 1. Experimental protocol. The task was divided into four types of blocks, each with event-related trials examining either perception (viewing) or imagery (imagining), with either face or moving dot stimuli. Perception and imagery blocks were parallel in design, presenting trials with a sequence of voice prompt, viewing or imagining the stimulus indicated by the prompt, a button press evaluation of the perceived or imagined stimulus, and an intertrial interval.

participate in this study, which was approved by the University of California, San Francisco Committee for Human Research. Four older and one younger participants' data were removed from the group analysis due to excessive motion artifacts. Additionally, data from one younger and one older participant were removed from analysis because the imagery selectivity indices was outside the 95th percentile for their group (Grubbs, 1969), leaving 14 younger (seven female) and 14 older (nine female) participants for final analysis. All participants had normal or corrected-to-normal vision and were screened to ensure they had no history of neurological, psychiatric, or vascular disease; were not depressed; and were not taking any psychotropic medications. All participants had a minimum of 12 years education.

Neuropsychological testing. Participants in the older age group were administered 13 neuropsychological tests of executive and memory function, and were found to be cognitively intact (i.e., within $2 \mathrm{SD}$ ) relative to normative values for age-matched controls. Neuropsychological testing was performed on a separate day from $\mathrm{fMRI}$ and included the following tests of general intellectual function: mini-mental status exam, verbal learning (California Verbal Learning Test-II), geriatric depression, visual-spatial function (copy of a modified Rey-Osterrieth figure), visual-episodic memory (memory for details of a modified ReyOsterrieth figure), visual-motor sequencing (Trail Making Test A and B), phonemic fluency (words beginning with the letter "D"), semantic fluency (animals), calculation ability (arithmetic), executive functioning (Stroop interference test), and working memory and incidental recall (backward digit span and digit symbol; Wechsler Adult Intelligence Scale-Revised).

Task design. Stimuli consisted of grayscale images of either famous faces or moving dots. Famous faces were used rather than unfamiliar faces to promote retention of faces for later recall during imagery (Ishai et al., 2002). The pool of face stimuli consisted of facial photographs of both contemporary and noncontemporary male and female celebrities. Participants were instructed to select five familiar faces (two male and three female or three male and two female) from among the pool to be presented during the main task. Motion stimuli consisted of a circular aperture of 290 dots $\left(0.08^{\circ} \times 0.08^{\circ}\right.$ each $)$ moving coherently in one of the four cardinal directions at $10^{\circ}$ per second. Stimuli subtended $8^{\circ}$ of visual angle centered at the fovea.

The experiment used two conditions-perception and imagery-in an event-related design (Fig. 1). The perception condition was divided into view-face and view-motion blocks, each requiring viewing of the respective stimuli. The imagery condition was divided into imagine-face and imagine-motion blocks, requiring visual mental imagery of the same category of stimuli presented in the corresponding perception condition. Thus, stimuli were presented in four different types of blocks (view-face, view-motion, imagine-face, and imagine-motion) and were not mixed within blocks. Trials in each block comprised a $1 \mathrm{~s}$ voice prompt announcing the name of the stimulus to be viewed or imagined (e.g., "Ein- stein" or "Left") followed immediately by a $4 \mathrm{~s}$ view or imagine period, rating period, and intertrial interval (ITI). Participants were instructed to keep their eyes open and focus on a fixation cross during the imagine trials. The stimulus for each trial was selected randomly from the corresponding pool of stimuli. To facilitate unbiased comparison of perceptual and imagery trials, voice prompts were included in the perceptual trials, even though they were redundant with visual stimuli. After viewing or imagining, participants were prompted by text to respond by pushing one of two buttons for the view condition (male or female for view face; horizontal or vertical movement for view motion) or one of four buttons for the imagine condition (rating vividness of imagery, 1-4, where 1 represents no imagery and 4 represents highly vivid imagery). Participants were instructed to respond as quickly as possible. This relatively simple task during the view condition was intended to promote attention to visual stimuli. The button press was followed by a $9 \mathrm{~s}$ ITI during which participants were instructed to clear their mind. Each block comprised 12 trials and was repeated four times, resulting in 48 repetitions of each condition-stimulus pair. Imagery blocks immediately followed the corresponding perception blocks to promote memory of stimuli. Before beginning the experiment, participants were given one practice block of each type outside of the scanner.

Data acquisition and analysis. All fMRI data were collected on a Siemens 3T MAGNETOM Trio with stimuli presented on an LCD monitor positioned behind the head of the participants and viewed using a mirror rigidly attached to a 12-channel head coil. Echo planar imaging data were acquired $\left(\mathrm{FA}=77^{\circ}, \mathrm{TE}=28 \mathrm{~ms}, \mathrm{TR}=2 \mathrm{~s}\right)$ with 29 interleaved axial slices and a $1.8 \times 1.8 \times 3 \mathrm{~mm}$ voxel size ( $\mathrm{FOV}=23 \mathrm{~cm}, 128 \times 128$ matrix). All preprocessing of the data were conducted in SPM5 (Wellcome Department of Imaging Neuroscience, London, England). Raw blood oxygen level-dependent (BOLD) data were corrected offline for slice-timing acquisition and motion artifacts. A $5 \mathrm{~mm}$ isotropic Gaussian smoothing kernel was applied before modeling the data. To aid in anatomical localizations of BOLD activity, high-resolution T1-MPRAGE images were acquired (voxel size, $1 \times 1 \times 1 \mathrm{~mm}$; FOV, $160 \times 240 \times 256 \mathrm{~mm}$; TR = $\left.2300 \mathrm{~ms}, \mathrm{TE}=3 \mathrm{~ms}, \mathrm{FA}=9^{\circ}\right)$.

All trial stages were modeled as events convolved with the canonical synthetic hemodynamic response function (SPM5; Wellcome Department of Imaging Neuroscience, London, England) and inserted in the general linear model (GLM). Regressor onset was time locked to the end of the voice cues in both the imagery and perception trials, corresponding to the onset of imagery and visual stimulus presentation, respectively. In addition, three translational $(x, y, z)$ and three rotational (pitch, roll, yaw) motion parameters were included in the GLM. The resulting regression vector yielded scalar beta weights corresponding to the relative changes in signal strength associated with a particular trial stage.

Region-of-interest localization and analysis. Two independent functional localizer tasks were used. The first task was used to identify the face-selective FFA (Kanwisher et al., 1997) regions and the second task to identify the motion-selective (V5) (Chawla et al., 1999) regions in the visual association cortex of each participant. For each localizer task, participants performed 10 blocks of a one-back task. Each block was $16 \mathrm{~s}$ in length. Blocks in the FFA localizer task included face stimuli interleaved with scene stimuli or fixation (rest). Blocks in the V5 localizer included motion stimuli interleaved with stationary gray dots. Blocked stimuli regressors were used to generate contrasts using a GLM approach (FFA from face $>$ scene contrasts; V5 from moving dots $>$ stationary dots contrasts). For the each localizer, ROIs were identified in native space as the cluster of 35 contiguous voxels with the highest $t$ values within the respective anatomical region (FFA, fusiform gyrus; V5, middle temporal gyrus), within each hemisphere. The ROI voxel extent was based on methodology from similar studies (Gazzaley et al., 2004; Rissman et al., 
2004) and was used to achieve a reasonable balance between regional specificity (diminished by the use of a larger cluster) and susceptibility to noise (a problem with smaller clusters).

ROIs were chosen for subsequent analysis based on functional localizer selectivity: right FFA ROIs were selected because across-participant averages of the face $>$ scene localizer contrast were greater in right FFA than left FFA, and left V5 ROIs were selected because across-participant averages of the moving dots $>$ stationary dots localizer contrast were greater in left V5 than right V5. Because imagery has been observed to preferentially activate regions in the nonperceptually dominant hemisphere (Ishai et al., 2000), we compared imagery selectivity in ROIs of both hemispheres. Collapsing across both younger and older data, imagery selectivity in left FFA $(0.98 \pm 0.42)$ was not significantly different from in right FFA $(1.1 \pm 0.40)\left(t_{26}=0.4 ; p>0.7\right)$. A similar analysis showed a trend toward greater imagery selectivity in left V5 $(0.92 \pm 0.42)$ compared with right V5 $(0.39 \pm 0.31)\left(t_{26}=1.85 ; p<0.1\right)$. Given that imagery selectivity was not greater in the contralateral hemisphere, ROIs from the perceptually dominant hemisphere were used in the analysis.

Normalization templates. To facilitate a valid, direct comparison of whole-brain data between the age cohorts (younger vs older), wholebrain data were normalized to a custom template, which was created according to the method delineated in Buckner et al. (2004). Anatomical images (MPRAGE) from the 14 younger and 14 older participants included in this study contributed to the template. Images from each age group were used to make an iteratively formed template unique for that age group. These two templates were averaged to create a new standard template. Subsequently, each subject's anatomical image (MPRAGE) was normalized to the new template to create subject-specific normalization parameters. Functional images (EPIs) were normalized using these parameters. Separately, the new template was normalized to MNI space to obtain a second set of transformation parameters. These newtemplate-to-MNI transformation parameters were applied to each subject's normalized images, resulting in a second transformation that was constant across participants. The second transformation affected cluster location, but not other cluster statistics, facilitating the communication of regional findings and validating the use of anatomical atlases.

Functional connectivity. Functional connectivity network maps were created for each subject as described previously, using the beta series connectivity analysis approach (Gazzaley et al., 2004; Rissman et al., 2004). For this analysis, a new GLM design matrix was constructed to model each trial with a separate regressor, resulting in separate beta values for each trial. For each condition, ROI (FFA and V5) beta values were then correlated across trials with beta values from every brain voxel, giving voxelwise covariate activity with each ROI for each participant in each condition. This procedure produces a whole-brain Pearson's $r$ value map for each subject and a Fisher's $r$-to- $z$ transformation was applied. Single-subject maps were subsequently normalized to the custom template in MNI space (voxel size, $2 \times 2 \times 2 \mathrm{~mm}$ ) and Gaussian smoothed ( 5 $\mathrm{mm}$ FWHM) for group analysis. Group contrast maps were created by using a permutation test (Nichols and Holmes, 2002). For all analyses, a single-voxel statistical threshold of $p<0.01$ was used. We performed Monte Carlo simulations using the AlphaSim function in the AFNI toolbox (Cox, 1996) to determine a cluster extent of 35 nearest-neighbor voxels to achieve a corrected $p<0.05$. One younger participant was removed from the functional connectivity analysis due to excessive noise throughout the map, resulting in inclusion of 13 younger participants in this analysis.

Postscan testing. A computerized version of the Continuous Visual Memory Test (CVMT) was administered to participants after the scanning session, followed by a postexperiment questionnaire and the Vividness of Mental Imagery Questionnaire (VVIQ) (Marks, 1973). In this standardized test, participants are asked to think of a series of objects/ activities and rate vividness on a five-point scale $(1=$ perfectly clear and vivid as normal vision; $5=$ no image at all).

\section{Results}

Non-neural assessment of visual imagery

All participants in both age groups reported successful generation of mental images in postexperiment questionnaires. Interest- ingly, subjective face imagery ratings were higher in older $(3.56 \pm$ $0.15)$ than younger $(2.96 \pm 0.14)\left(t_{26}=2.90 ; p<0.01\right)$ adults and subjective motion imagery ratings were also higher in older $(3.41 \pm 0.19)$ than younger $(2.7 \pm 0.16)$ adults $\left(t_{26}=2.77 ; p=\right.$ $0.01)$. However, average ratings in a standardized assessment of imagery vividness, the VVIQ, revealed no difference between younger $(2.09 \pm 0.29)$ and older $(2.29 \pm 0.29)$ adults $\left(t_{26}=0.59\right.$; $p>0.5)$. According to the test scale, these ratings represent a reasonably vivid level of imagery in both groups (Marks, 1973).

\section{Diminished visual cortex selectivity for imagery in aging}

We measured the BOLD signal during imagery and viewing tasks within the right FFA and left V5, which were the most robustly activated stimulus-selective regions identified using an independent localizer task (see Materials and Methods, above). For the perceptual task, the FFA was revealed to be selective for viewing faces (compared with viewing motion) and V5 for viewing motion (compared with viewing faces) in both younger (FFA: $t_{13}=$ $7.39, p<0.0001$; V5: $\left.t_{13}=5.89, p<0.0001\right)$ and older (FFA: $t_{13}=$ 6.92, $p<0.0001$; V5: $t_{13}=5.76, p<0.0001$ ) adults (Fig. $2 a$ ). There was no difference in perceptual selectivity between younger and older adults for either the FFA $\left(t_{26}=0.77, p>0.4\right)$ or V5 $\left(t_{26}=0.92, p>0.3\right)$. A different pattern was observed for the imagery task, such that both VAC regions were selective for stimulus category in younger adults (FFA: $t_{13}=3.78, p<0.01$; V5: $\left.t_{13}=2.75, p<0.05\right)$, while neither was selective in older adults (FFA: $t_{13}=0.49, p>0.6$; V5: $t_{13}=0.32, p>0.7$ ) (Fig. $2 b$ ). Interestingly, the absence of imagery selectivity in older adults was not the result of a failure to significantly activate the FFA and V5 during face and motion imagery, respectively, but rather an inability to activate these regions in a selective manner.

Next, we compared imagery selectivity between age groups. A direct comparison may be confounded by age-related differences in BOLD signal that do not reflect alterations in neural activity between the age groups (D'Esposito et al., 2003). Furthermore, although both groups as populations showed equivalent perceptual selectivity in these visual regions, individual differences in selectivity may influence comparisons of imagery selectivity between groups. Therefore, perceptual selectivity was used as a normalization factor in calculating an imagery selectivity index for each participant, as follows:

$$
\begin{gathered}
\text { MotionSelectivity }_{\mathrm{V} 5}=\frac{\text { Motion }_{\text {imagery }}-\text { Face }_{\text {imagery }}}{\text { Motion }_{\text {perception }}-\text { Face }_{\text {perception }}} \\
\text { FaceSelectivity }_{\mathrm{FFA}}=\frac{\text { Face }_{\text {imagery }}-\text { Motion }_{\text {imagery }}}{\text { Face }_{\text {perception }}-\text { Motion }_{\text {perception }}}
\end{gathered}
$$

Both face and motion imagery selectivity indices were significant in younger participants (faces: $t_{13}=3.79, p<0.005$; motion: $\left.t_{13}=3.09, p<0.01\right)$, but neither was significant in older adults (faces: $t_{13}=0.42, p>0.6$; motion: $t_{13}=0.39, p>0.7$ ). Importantly, both indices diminished significantly in older compared with younger adults (faces: $t_{26}=2.36, p<0.05$; motion: $t_{26}=$ $2.47, p<0.05$; Fig. $2 c$ ). These data indicate that aging is associated with a decrease in the selective manner that mental imagery activates category-selective areas of the VAC, independent of individual differences in perceptual selectivity.

To examine the relationship between these neural markers of imagery and the more commonly assessed index of imagery, subjective experience, we used the intraexperiment vividness of imagery rating designed to reflect the participants' introspective assessment of imagery strength on a trial-by-trial basis. To ac- 
Face Selective $\mathrm{ROI}$

a

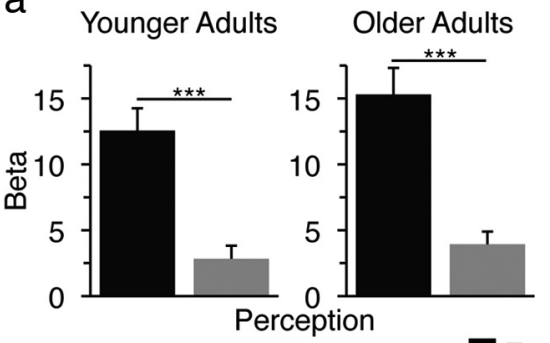

b

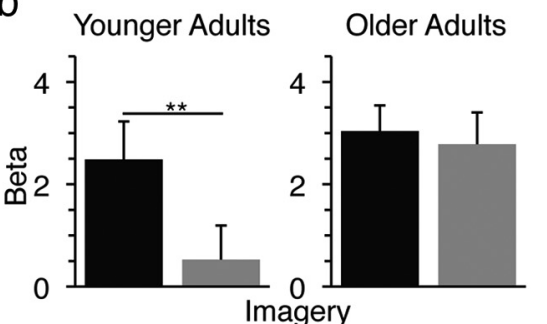

Faces
Motion Selective $\mathrm{ROI}$

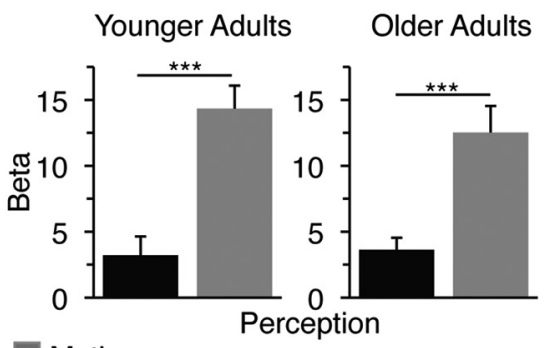

Motion

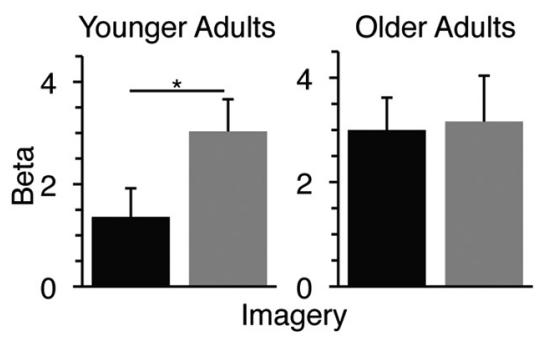

C 0.3 Imagery Selectivity Index

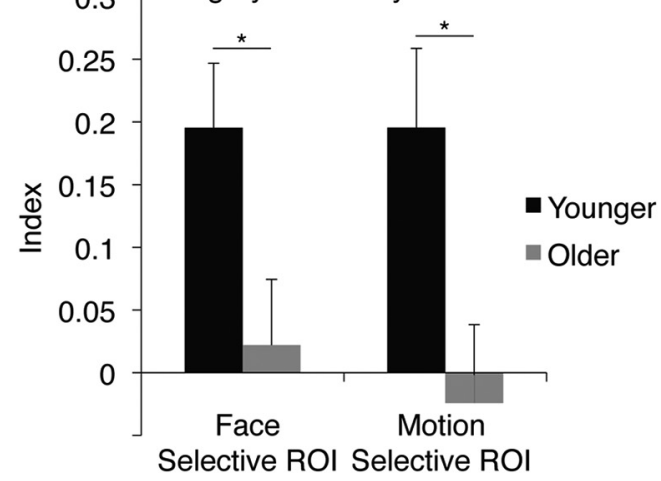

Figure 2. fMRI ROI analysis. $\boldsymbol{a}, \boldsymbol{b}$, Within-group comparisons of BOLD activity for both younger and older adults during perception in face-selective and motion-selective ROIs ( $\boldsymbol{a}$ ) and during imagery in face-selective and motion-selective ROls (b). $\boldsymbol{c}$, Acrossgroup comparisons of imagery selectivity index. Error bars are \pm SEM. ${ }^{* * *} p<0.0001 ;{ }^{* *} p<0.01 ;{ }^{*} p<0.05$.

cant correlation across all participants $(r=0.44 ; p<0.05$; Fig. 3$)$, demonstrating a relationship between subjective impressions of imagery vividness and neural measures of imagery fidelity. To evaluate the effect of age on this correlation, we calculated a partial correlation statistic, regressing for age. This also showed a significant correlation $(r=0.44, p<0.05)$, demonstrating that the correlation is maintained when removing the influence of age. Evaluating each age group separately revealed a trend in both the younger $(r=0.40, p=0.16)$ and older $(r=0.48$, $p=0.08)$ adults, with no significant difference between the groups (Fisher $z=$ $-0.26 ; p>0.05)$. This suggests that the relationship between subjective and objective measures of mental imagery is preserved in aging. We also assessed for the presence of a relationship between the neural index and VVIQ scores derived from a postexperiment questionnaire. No significant correlation was revealed for the study population or each age group analyzed independently. However, a study that compared VVIQ to fMRI activity during mental imagery in younger adults reported a trend in a correlation analysis between positive fMRI activation in visual cortical areas and the VVIQ (Amedi et al., 2005).

Age-related decreases in the selectivity of top-down imagery networks

Finding diminished selective activation of VAC in older adults during visual imagery, a process that occurs independent of bottom-up input, suggests that this deficit
lterations in top-down modulation. Given

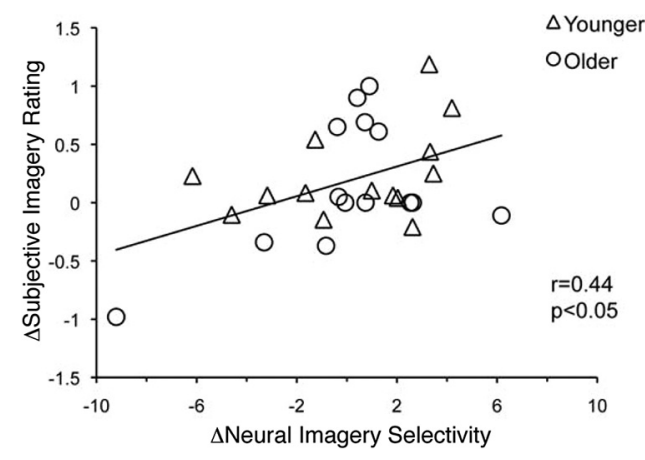

Figure 3. Relationship between subjective impressions of imagery and neural measures of imagery. Regression analysis between a difference score in the subjective imagery rating (i.e., face imagery ratings minus motion imagery ratings) and a difference score in the neural index of imagery selectivity (i.e., FFA imagery selectivity minus V5 imagery selectivity) across all participants.

complish this, an across-participant regression analysis was performed between a difference score in subjective imagery rating (i.e., face imagery ratings minus motion imagery ratings) and a difference score in neural imagery selectivity (i.e., FFA imagery selectivity minus V5 imagery selectivity). This showed a signifi- may be mediated by alterations in top-down modulation. Given
fMRI evidence that the PFC is functionally connected to VAC regions in a manner selective to imagery content (Mechelli et al., 2004), we hypothesized that altered selectivity of top-down PFC control underlies age-related deficits in mental imagery. To evaluate this, we first performed a beta series functional connectivity analysis of fMRI data using the FFA and V5 as seed regions to generate whole-brain imagery networks (Gazzaley et al., 2004; Rissman et al., 2004). This approach capitalizes on trial-by-trial variability to measure correlations in activity between spatially disparate brain regions, thus offering a powerful tool for assessing network interactions. We then contrasted whole-brain connectivity maps of congruent versus incongruent task-seed pairings to yield selective imagery networks (i.e., face-selective imagery network $=$ face imagery task with FFA seed $>$ motion imagery task with FFA seed; motion-selective imagery network = motion imagery task with V5 seed $>$ face imagery task with V5 seed). Finally, after normalizing these maps to a customized template generated using all participants' anatomical data, we contrasted them across age groups to explore for differences in the selectivity of imagery networks (Table 1). Consistent with our hypothesis, selective connectivity between the FFA and two PFC areas [i.e., the contralateral inferior frontal junction (IFJ) and superior frontal gyrus], were diminished in older compared with younger 
Table 1. Age-related changes in imagery selectivity networks between VAC and PFC

\begin{tabular}{llcrrr}
\hline Network & Area & Voxels & \multicolumn{1}{l}{$x$} & \multicolumn{1}{l}{$y$} & \multicolumn{1}{c}{$z$} \\
\hline Face-selective & L superior frontal gyrus* & 40 & -21 & 44 & 31 \\
& Linferior frontal junction* & 59 & -60 & 12 & 38 \\
& Ramygdala & 46 & 23 & -1 & -10 \\
& Rinferior temporal gyrus & 51 & 51 & -52 & -7 \\
Motion-selective & Rinferior frontal junction* & 54 & 48 & 23 & 39 \\
& R fusiform gyrus & 97 & 42 & -48 & -20 \\
& Roccipital gyrus & 115 & 46 & -66 & 12 \\
\hline
\end{tabular}

Significant (cluster: $p<0.05$; voxel: $p<0.01$; cluster extent: 35 voxels) clusters with positive connectivity in the younger $>$ older group contrast. The older $>$ younger contrast did not show any significant positive clusters. Face-selective imagery network $=$ right FFA seeded face-imagery $>$ motion-imagery. Motion-selective imagery network $=$ left V5 seeded motion-imagery $>$ face-imagery. L, Left; R, right.

*PFC areas depicted in Figure 3.
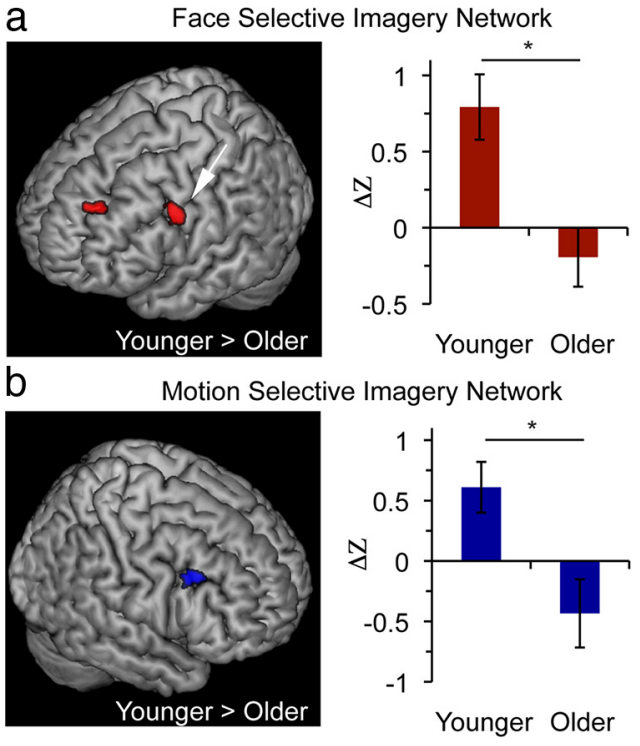

Figure 4. Reduced functional connectivity in older adults. $\boldsymbol{a}, \boldsymbol{b}$, Between-group contrasts (younger adults $>$ older adults) of imagery networks using face-selective seeds $(\boldsymbol{a})$ and motion-selective seeds $(\boldsymbol{b})$ (clusters $p<0.05$, corrected; only PFC areas depicted). Face imagery network contrast revealed regions in the right superior frontal gyrus (SFG) and IFJ ( $\boldsymbol{a}$; arrow, IFJ: $\mathrm{BA} 6 / 44 ;-60,12,39)$ and motion imagery network contrast revealed a region in the left IFJ (b; BA44; 48, 24, 40). Bar graphs depict connectivity measures in the IFJ regions found to be significant in cluster analysis to illustrate younger and older adult connectivity values that contribute to the results obtained by the whole-brain contrast. Coordinates in MNI. Error bars are \pm SEM. ${ }^{*} p<0.01$.

adults (Fig. 4a). Similarly, selective connectivity between V5 and a single PFC region, the contralateral IFJ, was diminished in older compared with younger adults (Fig. $4 b$ ). Several other regions exhibited greater connectivity in younger adults (Table 1), but no brain regions exhibited greater selective connectivity with the FFA or V5 seed in older versus younger adults.

\section{Imagery selectivity and visual memory}

Given the role of visual imagery in supporting memory performance (Kosslyn et al., 2001) and deficits in visual memory that occur with aging (Grady, 2008), we further hypothesized that a relationship may exist between visual imagery selectivity and visual memory in older adults. To evaluate this, we administered a test of visual memory outside of the scanner to all older participants [computerized version of the CVMT (Harker and Connolly, 2007)]. The CVMT is a test of visual recognition memory that uses complex, ambiguous shapes that are not easily susceptible to verbal labeling. Previous studies have demonstrated that CVMT performance $\left(d^{\prime}\right)$ is significantly lower in older than
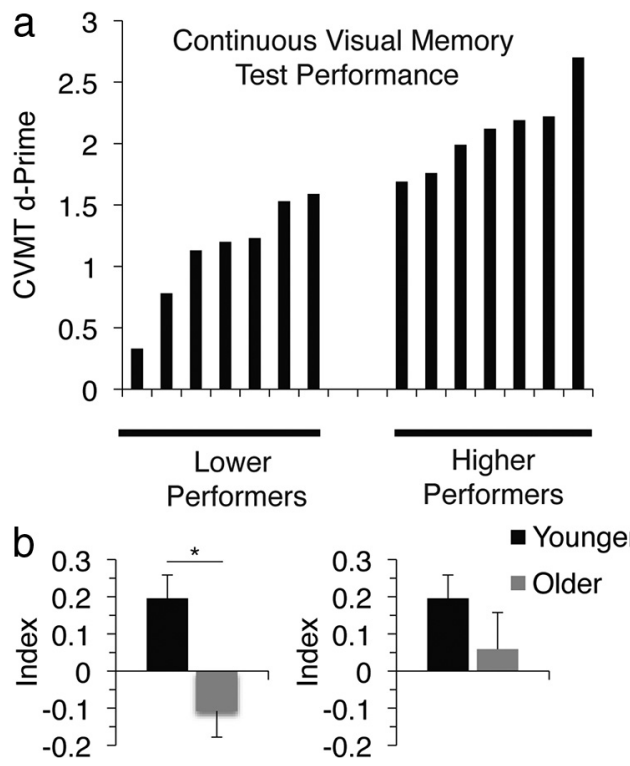

Figure 5. Relationship of imagery selectivity and visual memory in aging. $\boldsymbol{a}$, Subgroups of lower performing (left) and higher performing (right) older individuals (median split) on the CVMT, which was administered separately from the main task. $\boldsymbol{b}$, A significant motion imagery selectivity deficit is present in the lower-performing older subgroup (left), but not the higherperforming older subgroup (right). Error bars are \pm SEM. ${ }^{*} p<0.01$.

younger adults (Trahan et al., 1986). In the current study, memory performance subgroups of older participants [median split into lower $(n=7)$ and higher $(n=7)$ memory performance based on the CVMT $d^{\prime}$ ] exhibited differential motion imagery selectivity indices relative to younger adults. While the lower performing memory group demonstrated a significantly reduced motion imagery selectivity index compared with younger adults $\left(t_{19}=2.95, p<0.01\right)$, the higher performing older adults did not show a reduced index $\left(t_{19}=1.21, p=0.24\right)$ (Fig. 5). These results indicate that age-related differences in neural measures of imagery specificity were driven by older adults in the lower performing memory group, thus supporting a relationship between agerelated deficits in imagery selectivity and visual memory in the older population, consistent with the established relationship between mental imagery and memory in younger adults (Behrmann, 2000; Kosslyn et al., 2001). Of note, there was no relationship between the CVMT $d^{\prime}$ and the face selectivity index, possibly because the CVMT is a better correlate of motion imagery than face imagery [shape encoding has been localized to the dorsal visual pathway (Lehky and Sereno, 2007), associated with motion processing (Vaina et al., 2001)].

\section{Discussion}

The current study provides neurophysiological evidence that normal aging is associated with diminished selectivity in the activation of category-selective visual cortices during visual imagery. These neural data are indicative of degraded fidelity of cortical representations that support mental imagery. Broadening the implications of these findings, this effect occurred for both face and motion stimuli, suggesting that aging impacts imagery representations in both ventral and dorsal pathways. Critically, imagery selectivity was significantly reduced in older adults compared with younger adults despite correction for individual differences in VAC perceptual selectivity. This finding provides strong evidence in favor of an age-related deficit in visual imagery processes mediated by alterations in top-down modulation. This 
conclusion is supported by diminished selectivity of PFC-visual cortical networks during imagery. Moreover, only older adults with poorer visual memory performance demonstrated reduced imagery selectivity on the motion task compared with younger adults, suggesting that the imagery deficit is associated with agerelated memory impairment.

Although age differences in perceptual selectivity were not observed in the current study, it should be noted that decreased functional specialization of ventral visual cortical activations to faces, places, and words in older adults have been reported (Park et al., 2004). These changes have been interpreted as reflecting dedifferentiation of sensory cortices (Li et al., 2001) and have been shown to be associated with decreased distinctiveness of perceptual representations of faces (Goh et al., 2010). In the current study, the absence of evidence of diminished perceptual selectivity in aging is likely the result of differences in experimental design, e.g., different stimulus categories used. Faces and moving dots activate distinct dorsal and ventral visual pathways and may thus lack sensitivity to reveal perceptual differences compared with paradigms using stimuli represented in neighboring cortical regions. This raises the possibility that an age-related deterioration in imagery selectivity may be even more extreme for similar stimuli, which would also be impacted by dedifferentiation of perceptual representations.

Self report of successful mental imagery in a postexperiment questionnaire and high subjective imagery ratings during the experiment reveal that the older adults were engaging in the imagery task. This is supported by finding that the FFA and V5 are significantly active during the imagery task in older adults, just less selectively so. This alteration suggests that older adults exhibit impairment in selectively targeting top-down modulatory signals based on task goals. The presence of diminished selectivity in functional connectivity between the PFC and categoryselective visual cortices provides a neural mechanism for diminished selective activation during imagery. These findings may reflect dedifferentiation of PFC-VAC networks (Li et al., 2001), consistent with findings that neural specialization decreases in tasks under strong top-down control (Payer et al., 2006). The dedifferentiation hypothesis posits that distinctiveness of neural representations decrease with aging, leading to reduced regional process specificity ( $\mathrm{Li}$ et al., 2001). Thus, an age-related reduction in category-selective activation of VAC during imagery may reflect dedifferentiation of PFC control processes (Rajah and D'Esposito, 2005) under conditions where top-down modulation is required. This interpretation is consistent with evidence that cortical volumes of PFC regions correlate with behavioral measures of imagery in older adults (Briggs et al., 1999).

The results of the current study implicate the IFJ region of the PFC as a common area of age-related network changes for both motion and face imagery. This is consistent with a growing literature documenting the role of the IFJ in a diverse array of topdown control processes, including task switching, interference control, working memory, top-down modulation of visual feature processing, and expectancy-mediated neural biasing (Brass et al., 2005; Derrfuss et al., 2005; Bollinger et al., 2010; Zanto et al., 2010a,b). While the IFJ has not been explicitly implicated as a control region in mental imagery, a motion imagery study noted activations that lie within the coordinates of the IFJ (Goebel et al., 1998, labeled FEF) and two imagery studies using house, face, and chair stimuli document activations (Ishai et al., 2000) and effective connectivity nodes (Mechelli et al., 2004) in PFC regions immediately adjacent to the IFJ. These findings suggest that the IFJ may act as a control region for imagery representations local- ized to both ventral and dorsal pathways, consistent with recent evidence that networks between the IFJ and stimulus-selective visual areas mediate top-down control of both motion and color processing (Zanto et al., 2010b, 2011). The current results that aging decreases selectivity of IFJ-VAC functional connectivity for both motion and face imagery support this view, and further implicate the IFJ as a locus of altered top-down control associated with an age-related visual imagery deficit.

Finding a top-down control deficit in aging that occurs when external stimulation is absent may be related to impairment in top-down modulation that occurs in older adults when stimuli are present (Gazzaley et al., 2005, 2008; Zanto et al., 2010a). The PFC has a well documented role as a source of top-down modulatory influences of activity in sensory cortices both in the presence and absence of external stimuli (Gazzaley and D'esposito, 2007). Age-related alterations in PFC function and anatomy have been widely reported. The frontal lobes show the greatest atrophy in aging, the degree to which corresponds with cognitive deficits (Gunning-Dixon and Raz, 2003). Furthermore, complex patterns of activity changes in the PFC are seen in attention, episodic memory encoding, retrieval, and working memory in older adults (Grady et al., 1998; Cabeza et al., 2004; Dennis and Cabeza, 2008), underscoring the role of the PFC in top-down modulatory alterations in aging.

Recent studies have reported other examples of age-related processing deficits in the absence of external stimuli, which have been attributed to alterations in top-down modulation. For example, multitasking has been shown to disrupt the reactivation of interrupted internal representations during working memory maintenance to a greater degree in older adults (Clapp et al., 2011). Similarly, this has been associated with a deficit in functional connectivity between the PFC and VAC. Additionally, older adults exhibit diminished connectivity between PFC and VAC during expectation of a stimulus before its presentation (Bollinger et al., 2011). The current findings thus contribute to accumulating evidence of both stimulus-absent and stimuluspresent top-down modulation deficits in older adults, suggesting an impaired common top-down control mechanism resulting in a wide variety of cognitive deficits.

It has been reported in younger adults that decreased activation in auditory cortex occurs during visual imagery, and this may bolster the fidelity of mental imagery by filtering irrelevant sensory information (Amedi et al., 2005). This suggests the possibility that top-down suppression deficits that occur in older adults (Gazzaley et al., 2005, 2008) may contribute to observed age-related imagery deficits. However, the design of the current study was not ideal to assess auditory cortex deactivation during visual imagery, since an auditory cue immediately preceded each imagery event. A potential role of the age-related suppression deficit in impairment of mental imagery processes will be addressed in future studies.

The finding that only older adults with lower visual memory performance exhibited reduced imagery selectivity compared with younger adults suggests the possibility that memory impairment may be causally related to the imagery deficit. This result is consistent with a relationship between imagery and memory reported in younger adults (Johnson et al., 2009; Wais et al., 2010). Additionally, it extends previous behavioral evidence that an increase in the time it takes older adults to complete a mental scanning task correlates with decreased visual memory performance (Palladino and De Beni, 2003; Iachini et al., 2005).

In conclusion, this study provides the first neurophysiological evidence of a visual imagery deficit in normal aging and docu- 
ments the utility of using functional neuroimaging to evaluate changes in the selectivity of cortical representations and associated neural networks of imagery processes. It also highlights the broad range of age-related cognitive deficits that are associated with alterations in top-down modulation.

\section{References}

Amedi A, Malach R, Pascual-Leone A (2005) Negative BOLD differentiates visual imagery and perception. Neuron 48:859-872.

Behrmann M (2000) The mind's eye mapped onto the brain's matter. Curr Dir Psychol Sci 9:50-54.

Bollinger J, Rubens MT, Zanto TP, Gazzaley A (2010) Expectation-driven changes in cortical functional connectivity influence working memory and long-term memory performance. J Neurosci 30:14399-14410.

Bollinger J, Rubens MT, Masangkay E, Kalkstein J, Gazzaley A (2011) An expectation-based memory deficit in aging. Neuropsychologia 49:14661475.

Brass M, Derrfuss J, Forstmann B, von Cramon DY (2005) The role of the inferior frontal junction area in cognitive control. Trends Cogn Sci 9:314-316.

Briggs SD, Raz N, Marks W (1999) Age-related deficits in generation and manipulation of mental images. I. The role of sensorimotor speed and working memory. Psychol Aging 14:427-435.

Buckner RL, Head D, Parker J, Fotenos AF, Marcus D, Morris JC, Snyder AZ (2004) A unified approach for morphometric and functional data analysis in young, old, and demented adults using automated atlas-based head size normalization: reliability and validation against manual measurement of total intracranial volume. Neuroimage 23:724-738.

Chawla D, Rees G, Friston KJ (1999) The physiological basis of attentional modulation in extrastriate visual areas. Nat Neurosci 2:671-676.

Clapp WC, Rubens MT, Sabharwal J, Gazzaley A (2011) Neural basis for the negative impact of multitasking on working memory in older adults. Proc Natl Acad Sci U S A 108:7212-7217.

Cox RW (1996) AFNI: software for analysis and visualization of functional magnetic resonance neuroimages. Comput Biomed Res 29:162-173.

Craik FI, Dirkx E (1992) Age-related differences in three tests of visual imagery. Psychol Aging 7:661-665.

De Beni R, Pazzaglia F, Gardini S (2007) The generation and maintenance of visual mental images: evidence from image type and aging. Brain Cogn 63:271-278.

Derrfuss J, Brass M, Neumann J, von Cramon DY (2005) Involvement of the inferior frontal junction in cognitive control: meta-analyses of switching and Stroop studies. Hum Brain Mapp 25:22-34.

D'Esposito M, Deouell LY, Gazzaley A (2003) Alterations in the BOLD fMRI signal with ageing and disease: a challenge for neuroimaging. Nat Rev Neurosci 4:863-872.

Dror IE, Kosslyn SM (1994) Mental imagery and aging. Psychol Aging 9:90-102.

Gazzaley A, D'esposito M (2007) Unifying prefrontal cortex function: executive control, neural networks and top-down modulation. In: The human frontal lobes (Miller B, Cummings J, eds). New York: Guildford Publications.

Gazzaley A, Rissman J, D’Esposito M (2004) Functional connectivity during working memory maintenance. Cogn Affect Behav Neurosci 4:580-599.

Gazzaley A, Cooney JW, Rissman J, D’Esposito M (2005) Top-down suppression deficit underlies working memory impairment in normal aging. Nat Neurosci 8:1298-1300.

Gazzaley A, Clapp W, Kelley J, McEvoy K, Knight RT, D’Esposito M (2008) Age-related top-down suppression deficit in the early stages of cortical visual memory processing. Proc Natl Acad Sci U S A 105:13122-13126.

Goebel R, Khorram-Sefat D, Muckli L, Hacker H, Singer W (1998) The constructive nature of vision: direct evidence from functional magnetic resonance imaging studies of apparent motion and motion imagery. Eur J Neurosci 10:1563-1573.

Goh JO, Suzuki A, Park DC (2010) Reduced neural selectivity increases fMRI adaptation with age during face discrimination. Neuroimage $51: 336-344$

Grady CL (2008) Cognitive neuroscience of aging. Ann N Y Acad Sci 1124:127-144.
Grubbs FE (1969) Procedures for detecting outlying observations in samples. Technometrics 11:1-21.

Gunning-Dixon FM, Raz N (2003) Neuroanatomical correlates of selected executive functions in middle-aged and older adults: a prospective MRI study. Neuropsychologia 41:1929-1941.

Harker KT, Connolly JF (2007) Assessment of visual working memory using event-related potentials. Clin Neurophysiol 118:2479-2488.

Iachini T, Poderico C, Ruggiero G, Iavarone A (2005) Age differences in mental scanning of locomotor maps. Disabil Rehabil 27:741-752.

Ishai A, Ungerleider LG, Haxby JV (2000) Distributed neural systems for the generation of visual images. Neuron 28:979-990.

Ishai A, Haxby JV, Ungerleider LG (2002) Visual imagery of famous faces: effects of memory and attention revealed by fMRI. Neuroimage 17:1729_ 1741.

Johnson JD, McDuff SG, Rugg MD, Norman KA (2009) Recollection, familiarity, and cortical reinstatement: a multivoxel pattern analysis. Neuron 63:697-708.

Kanwisher N, McDermott J, Chun MM (1997) The fusiform face area: a module in human extrastriate cortex specialized for face perception. J Neurosci 17:4302-4311.

Kosslyn SM, Thompson WL, Alpert NM (1997) Neural systems shared by visual imagery and visual perception: a positron emission tomography study. Neuroimage 6:320-334.

Kosslyn SM, Ganis G, Thompson WL (2001) Neural foundations of imagery. Nat Rev Neurosci 2:635-642.

Lehky SR, Sereno AB (2007) Comparison of shape encoding in primate dorsal and ventral visual pathways. J Neurophysiol 97:307-319.

Li SC, Lindenberger U, Sikström S (2001) Aging cognition: from neuromodulation to representation. Trends Cogn Sci 5:479-486.

Marks DF (1973) Visual imagery differences in the recall of pictures. Br J Psychol 64:17-24.

Mechelli A, Price CJ, Friston KJ, Ishai A (2004) Where bottom-up meets top-down: neuronal interactions during perception and imagery. Cereb Cortex 14:1256-1265.

Nichols TE, Holmes AP (2002) Nonparametric permutation tests for functional neuroimaging: a primer with examples. Hum Brain Mapp 15:1-25.

O'Craven KM, Kanwisher N (2000) Mental imagery of faces and places activates corresponding stimulus-specific brain regions. J Cogn Neurosci 12:1013-1023.

Palladino P, De Beni R (2003) When mental images are very detailed: image generation and memory performance as a function of age. Acta Psychologica 113:297-314

Park DC, Polk TA, Park R, Minear M, Savage A, Smith MR (2004) Aging reduces neural specialization in ventral visual cortex. Proc Natl Acad Sci U S A 101:13091-13095.

Payer D, Marshuetz C, Sutton B, Hebrank A, Welsh RC, Park DC (2006) Decreased neural specialization in old adults on a working memory task. Neuroreport 17:487-491.

Rajah MN, D'Esposito M (2005) Region-specific changes in prefrontal function with age: a review of PET and fMRI studies on working and episodic memory. Brain 128:1964-1983.

Rissman J, Gazzaley A, D'Esposito M (2004) Measuring functional connectivity during distinct stages of a cognitive task. Neuroimage 23:752-763.

Saimpont A, Pozzo T, Papaxanthis C (2009) Aging affects the mental rotation of left and right hands. PLoS One 4:e6714.

Trahan DE, Larrabee GJ, Levin HS (1986) Age-related differences in recognition memory for pictures. Exp Aging Res 12:147-150.

Vaina LM, Solomon J, Chowdhury S, Sinha P, Belliveau JW (2001) Functional neuroanatomy of biological motion perception in humans. Proc Natl Acad Sci U S A 98:11656-11661.

Wais PE, Rubens MT, Boccanfuso J, Gazzaley A (2010) Neural mechanisms underlying the impact of visual distraction on retrieval of long-term memory. J Neurosci 30:8541-8550.

Zanto TP, Toy B, Gazzaley A (2010a) Delays in neural processing during working memory encoding in normal aging. Neuropsychologia 48:13-25.

Zanto TP, Rubens MT, Bollinger J, Gazzaley A (2010b) Top-down modulation of visual feature processing: the role of the inferior frontal junction. Neuroimage 53:736-745.

Zanto TP, Rubens MT, Thangavel A, Gazzaley A (2011) Causal role of the prefrontal cortex in top-down modulation of visual processing and working memory. Nat Neurosci 14:656-661. 